\title{
PICK1 deficiency causes male infertility in mice by disrupting acrosome formation
}

\author{
Nan Xiao, ${ }^{1}$ Chuen Kam, ${ }^{1}$ Chong Shen, ${ }^{1}$ Wenying Jin, ${ }^{1}$ Junqi Wang, ${ }^{2}$ \\ Kwong Man Lee, ${ }^{3}$ Liwen Jiang, ${ }^{2}$ and Jun $\mathrm{Xia}^{1}$
}

\begin{abstract}
1Department of Biochemistry, The Hong Kong University of Science and Technology, Clear Water Bay, Kowloon, Hong Kong, People's Republic of China. 2Department of Biology and ${ }^{3} \mathrm{Li}$ Ka Shing Institute of Health Sciences, Faculty of Medicine, The Chinese University of Hong Kong,
\end{abstract} Shatin, New Territories, Hong Kong, People's Republic of China.

\begin{abstract}
Protein interacting with $\mathrm{C}$ kinase 1 (PICK1) is a peripheral membrane protein involved in protein trafficking, a function that has been well characterized in neurons. Here, we report that male mice deficient in PICK1 are infertile and have a phenotype resembling the human disease globozoospermia. The primary defect in the testes of Pick1-knockout mice was fragmentation of acrosomes in the early stages of spermiogenesis. This fragmentation was followed by defects in nuclear elongation and mitochondrial sheath formation, leading to round-headed sperm, reduced sperm count, and severely impaired sperm motility. We found that PICK1 interacted with Golgi-associated PDZ- and coiled-coil motif-containing protein (GOPC) and the primary catalytic subunit of protein kinase $2\left(\mathrm{CK} 2 \alpha^{\prime}\right)$, proteins whose deficiencies lead to globozoospermia in mice. PICK1 was highly expressed in round spermatids and localized to Golgi-derived proacrosomal granules. GOPC colocalized with PICK1 in the Golgi region and facilitated formation of PICK1-positive clusters. Furthermore, there was an increase in apoptosis in the seminiferous tubules of Pick1 $1^{-/}$mice, a phenotype also seen in CK2 $\alpha^{\prime}$-deficient mice. Our results suggest that PICK1 is involved in vesicle trafficking from the Golgi apparatus to the acrosome and cooperates with other proteins such as GOPC and $\mathrm{CK} 2 \alpha^{\prime}$ in acrosome biogenesis.
\end{abstract}

\section{Introduction}

Protein interacting with C kinase 1 (PICK1) is a peripheral membrane protein involved in protein trafficking. PICK1 was initially identified as a protein kinase $\mathrm{C}$-interacting protein from a yeast two-hybrid screen (1). Subsequently, many proteins have been found to interact with PICK1 (2). The majority of these proteins are membrane proteins, such as glutamate receptors, dopamine transporter, Eph receptors, and acid-sensing ion channels (3-8). These interactions usually occur between the $\mathrm{C}$ termini of the membrane proteins and PICK1's postsynaptic density 95, discs large, and zonula occludens-1 (PDZ) domain, a well-characterized protein-protein interaction module. In most cases, PICK1 regulates the subcellular localization or cell-surface expression of its PDZ domain-binding partners.

Studies of PICK1's role in $\alpha$-amino-3-hydroxy-5-methyl-4-isoxazolepropionic acid (AMPA) receptor trafficking have provided much information that helps us to understand PICK1's function. The AMPA receptor is a subtype of glutamate receptor that mediates the majority of excitatory synaptic transmission in the brain (9). The role of PICK1 has been extensively studied in AMPA receptor trafficking because of its implication in synaptic plasticity, a cellular model of learning and memory (10). PICK1 was found to interact specifically with the $\mathrm{C}$ termini of AMPA receptor subunits

Conflict of interest: The authors have declared that no conflict of interest exists.

Nonstandard abbreviations used: AMPA, $\alpha$-amino-3-hydroxy-5-methyl-4-isoxazolepropionic acid; BAR, Bin/amphiphysin/Rvs; BFA, brefeldin A; CK2 $\alpha^{\prime}$, the primary catalytic subunit of protein kinase 2; DAB, diaminobenzidine tetrahydrochloride; GOPC, Golgi-associated PDZ- and coiled-coil motif-containing protein; GST, glutathione $S$-transferase; Hrb, HIV-1 Rev binding protein; ICA69, islet cell autoantigen $69 \mathrm{kDa}$; PDZ, postsynaptic density 95, discs large, and zonula occludens-1; PICK1, protein interacting with $\mathrm{C}$ kinase 1 ; TEM, transmission electron microscope/microscopy; ZPBP, zona pellucida binding protein.

Citation for this article: J. Clin. Invest. 119:802-812 (2009). doi:10.1172/JCI36230.
GluR2 and GluR3 via its PDZ domain $(4,5)$. Through its interaction with AMPA receptors, PICK1 induces formation of AMPA receptor clusters in heterologous cells and targets AMPA receptors to synapses in neurons. PICK1 was also found to reduce the surface expression of AMPA receptors (11). PICK1's roles in synaptic targeting and surface expression of AMPA receptors were found to be important to synaptic plasticity, as perturbing the interaction between PICK1 and AMPA receptors impairs synaptic plasticity (12-14). PICK1's role in AMPA receptor trafficking and synaptic plasticity has been further supported by data from Pick1-knockout mice, which were found to have defects in AMPA receptor trafficking and synaptic plasticity $(15,16)$.

Recent studies have begun to reveal the molecular mechanism underlying PICK1-regulated protein trafficking. PICK1 has 2 major domains. In addition to its PDZ domain, PICK1 also has a Bin/amphiphysin/Rvs (BAR) domain. BAR domains are crescentshaped dimers that bind and facilitate the formation of membrane curvatures to form budding vesicles (17). PICK1's BAR domain binds to negatively charged lipids, mainly phosphoinositides (11). Lipid binding-deficient PICK1 loses its ability to regulate AMPA receptor trafficking and impairs expression of synaptic plasticity. In Pick1-knockout mice, the wild-type PICK1 cDNA was found to rescue the synaptic plasticity defects, while the lipid binding-deficient PICK1 cDNA failed to do so (15). Although PICK1's BAR domain is capable of forming homodimers, the majority of PICK1 in the brain actually forms heterodimers with another BAR domain-containing protein, islet cell autoantigen $69 \mathrm{kDa}$ (ICA69) (18), an autoantigen first identified in patients with type 1 diabetes (19). Formation of heterodimers with ICA69 reduces synaptic targeting of PICK1 and surface expression of AMPA receptors. These results suggest that PICK1's BAR domain, via its binding to membrane curvatures, may tether PICK1 PDZ domain-binding partners, such as AMPA receptors, as cargo to trafficking vesicles. 
A

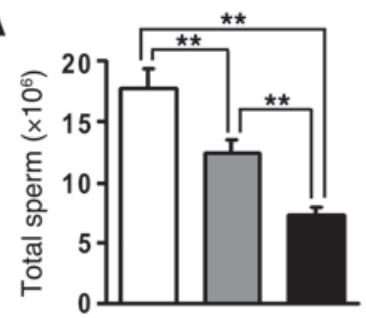

B
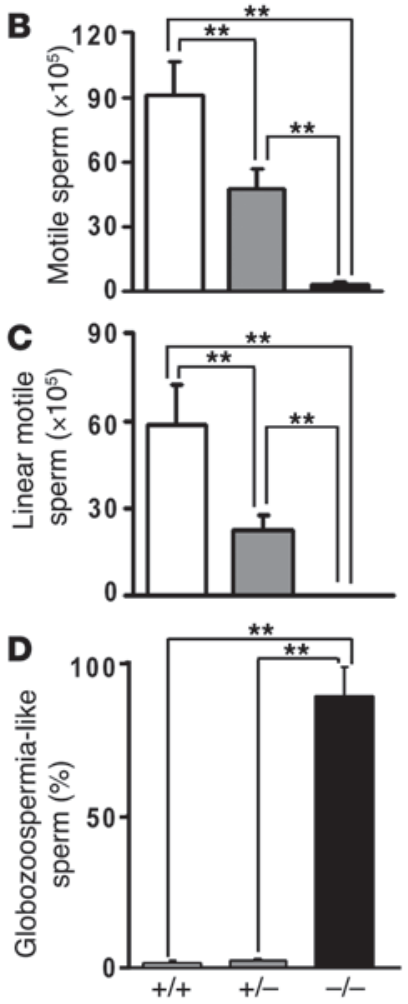

E

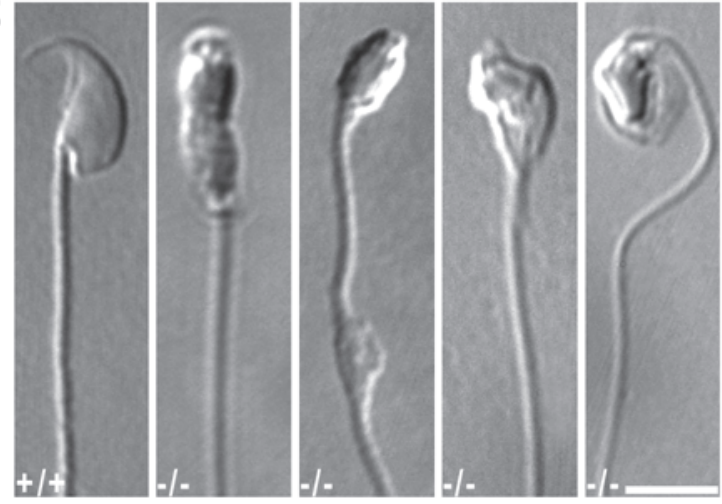

$\mathbf{F}$
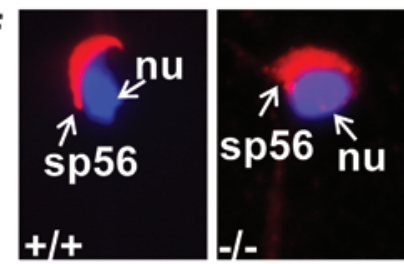

G
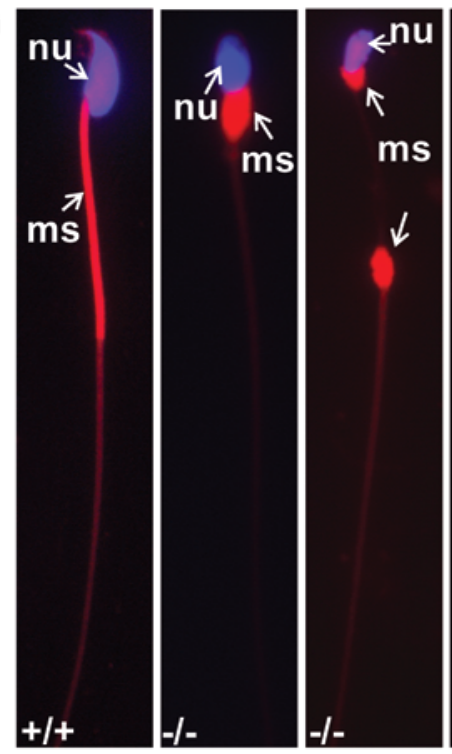
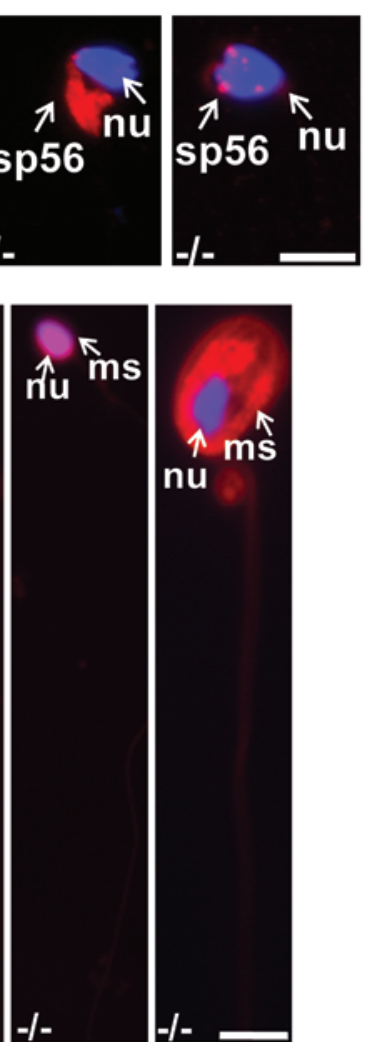

Figure 1

Decreased sperm number and abnormal sperm morphology in Pick $1^{-1-}$ mice. (A) Total number of sperm from a single cauda epididymis: wild-type $\left(\right.$ Pick $\left.1^{+/+}\right), 17.69 \times 10^{6}$ $\pm 1.62 \times 10^{6} ;$ Pick1+-, $12.41 \times 10^{6}$ $\pm 1.10 \times 10^{6} ;$ Pick1 $^{-/}, 7.24 \times 10^{6} \pm$ $0.81 \times 10^{6} ;$ mean \pm SEM, $n=10$, ${ }^{* *} P<0.01$. (B) Motile sperm number: Pick1+l+, $91.03 \times 10^{5} \pm$ $15.5 \times 10^{5} ;$ Pick1+/-, $47.68 \times 10^{5}$ $\pm 9.32 \times 10^{5} ;$ Pick1 $1^{-/}, 3.38 \times 10^{5} \pm$ $1.21 \times 10^{5}$. (C) Linear motile sperm number: Pick1+/+, $58.80 \times 10^{5} \pm$ $13.59 \times 10^{5} ;$ Pick1+/-, $22.13 \times 10^{5} \pm$ $5.55 \times 10^{5}$; Pick1-1-, 0. (D) The percentage of globozoospermia-like sperm: Pick $1^{+/+}, 1.57 \% \pm 0.81 \%$; Pick1+-, 2.26\% \pm 0.73\%; Pick1-1-, $88.69 \% \pm 9.57 \%(n=3)$. (E) Morphology of unfixed sperm. Sperm from Pick $1^{-/}$mice lose the typical hook-shaped head of normal sperm; instead, they have round or irregular ball-like heads. In addition, defects in the tail can also be seen in sperm from Pick $1^{-1-}$ mice. (F) Immunostaining of acrosome matrix protein sp56 (red) and nucleus (nu, blue) in sperm. The acrosomes from Pick 1-/- mice fail to acquire the crescent moon-shaped structure, are frequently fragmented, and are located in the wrong position. (G) Immunostaining of the mitochondrial sheath (ms, red) and nucleus in sperm. Mitochondrial sheaths in Pick ${ }^{-1}$ mice display various defects, including (left to right) abnormal sperm with aggregated mitochondrial sheaths, split mitochondrial sheaths, the mitochondrial sheath overlapping with the round nucleus, and the mitochondrial sheath wrapping around the round nucleus. Scale bars: $5 \mu \mathrm{m}$.
The transition from the PICK1-ICA69 heterodimer to the PICK1PICK1 homodimer may determine the destination of these trafficking vesicles. Since the PDZ domain of PICK1 binds to more than 40 proteins, it is likely that PICK1 may regulate the trafficking of these proteins in similar ways (2).

PICK1 is expressed not only in the brain, but also in many other tissues, with relatively high levels in the testes and the pancreas (18). The functions of PICK1 outside the brain remain largely unknown. Here we report that, surprisingly, male Pick1-knockout mice are completely infertile. Detailed analysis reveals that Pick1knockout mice have round-headed sperm with malformed acrosomes and abnormal nuclear shapes, characteristics of the sperm found in a human disease called globozoospermia (20). PICK1 is localized at the Golgi-derived proacrosomal granules in spermatids, and the globozoospermia phenotype of Pick1-knockout mice is likely a consequence of abnormal trafficking of proacrosomal granules that leads to impaired formation of acrosomes.

\section{Results}

Abnormalities of the sperm in Pick1 1/- mice resemble those found in human globozoospermia. Pick1-knockout mice appear to be grossly normal $(15,16)$. Although male Pick $1^{-/}$mice copulate normally, they are completely infertile. We determined the numbers and sizes of litters and found that when Pick $1^{+/-}$male mice were mated with Pick $1^{+/-}$ female mice over a 6-month period, the fertility rate was comparable to that of wild-type mice (average litter size [mean \pm SEM]: male Pick $1^{+/+} \times$female Pick $1^{+/+}, 7.3 \pm 0.4$, male Pick $1^{+/-} \times$female Pick $1^{+/-}$, $7.1 \pm 0.5$; average litter number: male Pick $1^{+/+} \times$female Pick $1^{+/+}$, $4.3 \pm 0.2$, male Pick $1^{+/} \times$female Pick $\left.1^{+/-}, 4.3 \pm 0.2 ; n=20\right)$. On the other hand, when Pick1 $1^{-/}$males were mated with either wild-type or Pick $1^{+/-}$female mice, no offspring were obtained.

To investigate how PICK1 deficiency leads to male infertility, we first examined the sperm of Pick $1^{-/-}$mice. The total number of sperm from the cauda epididymis of adult Pick $1^{-/-}$mice $\left(7.24 \times 10^{6}\right.$ $\left.\pm 0.81 \times 10^{6}\right)$ was significantly smaller than that of the wild-type 
A
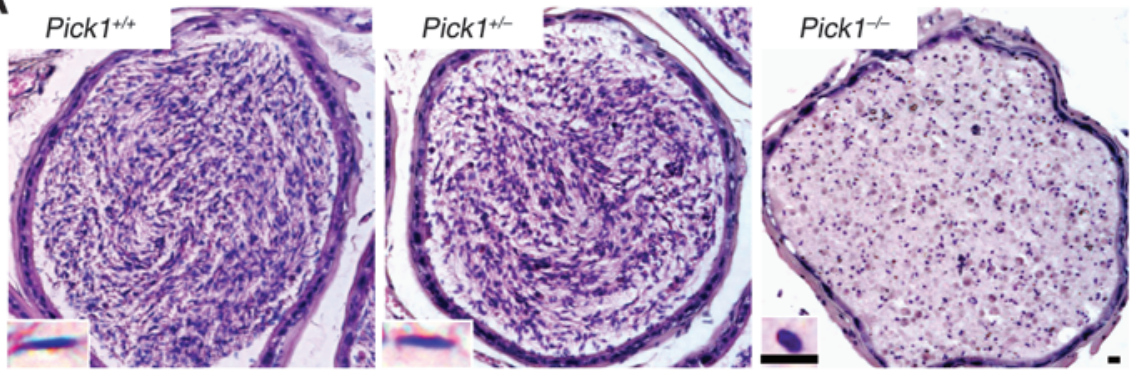

B

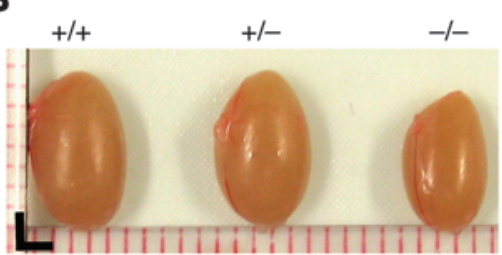

C

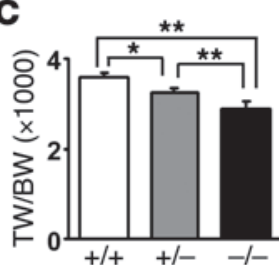

D

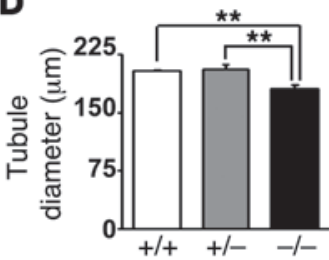

$\mathbf{E}$
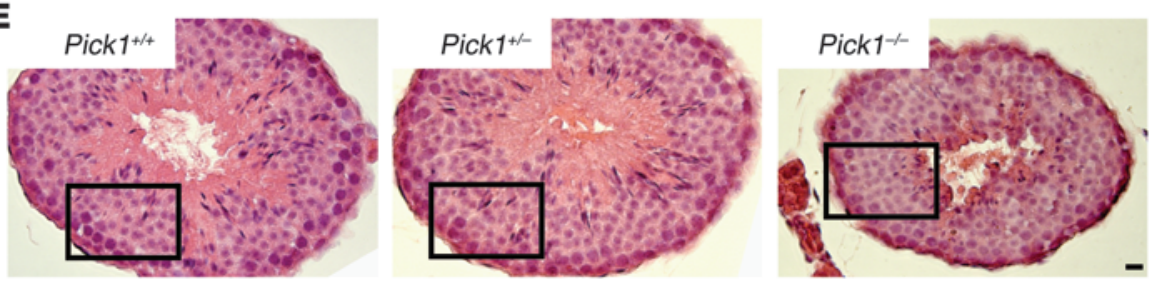
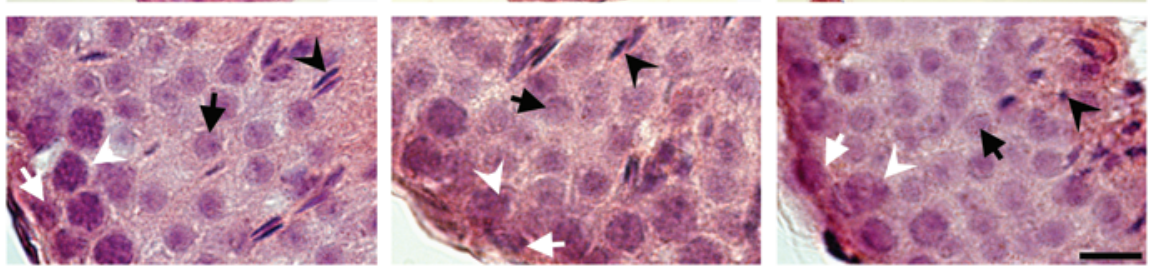

Figure 2

Abnormal spermiogenesis in Pick $1^{-/}$mice. (A) H\&E staining of the epididymis. The number of sperm in Pick $1^{-1-}$ mice is lower than that in Pick $1^{+/+}$mice in the cross sections of the cauda epididymis. Insets: Enlarged views of the heads of the sperm, showing that the sperm from Pick $1^{-1-}$ mice are round-headed. Scale bars: $10 \mu \mathrm{m}$. (B) The testes of Pick $1^{-1-}$ mice are smaller than those of Pick $1^{+/+}$or Pick1+/- mice. Scale bar: $2 \mathrm{~mm}$. (C) Normalized testis weights (Testis weight (TW) $\times 1000 /$ BW) of Pick1-/- mice are significantly lower than those of the Pick $1^{+/+}$or Pick1 $1^{+-}$mice. Pick1+/+, $3.58 \pm 0.11$; Pick1+/-, $3.25 \pm 0.09$; Pick1-/, $2.88 \pm 0.19 ; n=10$. Data are presented as mean \pm SEM; ${ }^{*} P<0.05,{ }^{* *} P<0.01$. (D) The diameter of the seminiferous tubules in Pick1-1mice is smaller than that of the Pick $1^{+/+}$and Pick $1^{+/-}$mice. Pick $1^{+/+}, 203.7 \pm 1.13 \mu \mathrm{m}$; Pick $1^{+/-}$, $206.2 \pm 5.2 \mu \mathrm{m} ;$ Pick $1^{-1-}, 181.3 \pm 4.2 \mu \mathrm{m} ; n=50$. (E) H\&E staining of testis. The lumens of seminiferous tubules in Pick1-- mice are slightly larger than those in wild-type mice. The morphology of spermatogonia (white arrows), spermatocytes (white arrowheads), and round spermatids (black and the heads of the sperm in Pick $1^{-/}$mice are round, in contrast to the hook-shaped heads found in Pick 1+/+ and Pick1+/- mice (black arrowheads). The bottom row shows higher-magnification views of the boxed regions in the top panels. Scale bar: $10 \mu \mathrm{m}$. arrows) is similar in the 3 genotypes. However, there are fewer mature sperm in the Pick $1^{-1-}$ mice,

while the sperm from wild-type mice were hook-shaped (Figure 1E, brightfield images). The defects were clearly revealed by staining with DAPI, which labels the nucleus, and with sp56, which marks the acrosome. The acrosome is a specialized secretory structure located in the head of mammalian sperm (21-23). It contains various hydrolyzing enzymes that are released when the sperm comes into contact with the zona pellucida of an egg, and these enzymes facilitate the sperm's penetration and fusion with the egg. As shown in Figure 1F, the acrosomes of the sperm from Pick $1^{-/-}$mice failed to acquire the typical crescent moon shape, with defects including mislocalization, deformation, and fragmentation. The mitochondrial sheath, which is responsible for sperm movement, also exhibited various defects in the sperm of Pick1 $1^{-/}$mice. Immunostaining with cytochrome oxidase subunit I, which marks mitochondria, revealed that the mitochondria in the sperm of Pick1 $1^{-/}$ mice have a variety of defects, including aggregating near the deformed nucleus, splitting into two separate aggregates, overlaying with the deformed nucleus, and in some cases wrapping around the deformed nucleus (Figure 1G). Quantification results indicated that nearly $90 \%$ of the sperm from the cauda epididymis of Pick $1^{-/-}$mice were round-headed with abnormal acrosomes, round nuclei, and abnormal mitochondrial sheaths (Figure 1D). In contrast, abnormal sperm with all 3 defects were rarely seen in wild-type or heterozygous mice. These abnormalities are reminiscent of the defects seen in globozoospermia, a human infertility disorder characterized by round-headed sperm with deformed nuclei, abnormal acrosomes, and malformed mitochondrial sheaths (20).

Abnormal spermiogenesis in Pick1 $1^{-/}$mice. To determine the causes of the abnormalities in the sperm of $\mathrm{Pick}^{-/-}$mice, we examined the morphology of the sperm in the male reproductive tract. $\mathrm{H} \& \mathrm{E}$ staining revealed that there were fewer $\left(17.69 \times 10^{6} \pm 1.62 \times 10^{6}\right)$ and Pick1 $1^{+/}$mice $\left(12.41 \times 10^{6} \pm 1.10 \times 10^{6}\right)$ (Figure 1A). Sperm motility was even more severely affected by the PICK1 deficiency. The number of motile sperm from Pick1-1- mice was less than $4 \%$ of that from their wild-type littermates, and none of the sperm from Pick1 $1^{-/}$mice exhibited rapid progressive linear motility (Figure 1, B and C).

When examining the morphology of the sperm from the caudal epididymis, we observed that a large number of sperm from Pick $1^{-1}$ mice had abnormal heads resembling irregularly shaped balls, sperm in the cross sections of the cauda and caput epididymis of Pick1 $1^{-/-}$mice (Figure 2A; only the cauda epididymis is shown). In addition, the sperm in the epididymis of Pick1 $1^{-/}$mice are round rather than of the typical hooked shape found in their wild-type and heterozygous littermates (Figure 2A, insets). The finding that abnormal sperm are present throughout the epididymis suggests that the defects may originate from the testes. The testes of Pick $1^{-/-}$ mice appeared to be normal upon gross examination. However, they were found to be slightly smaller than those of wild-type mice 
A

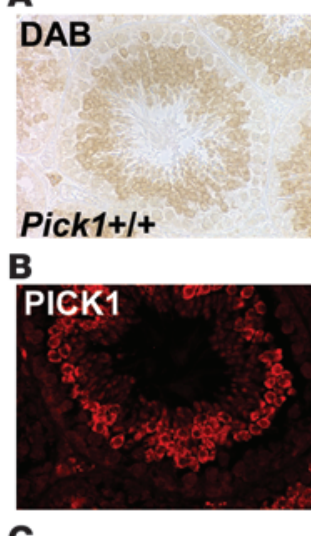

C
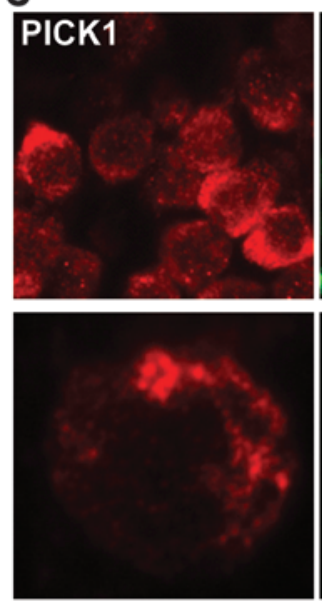

D

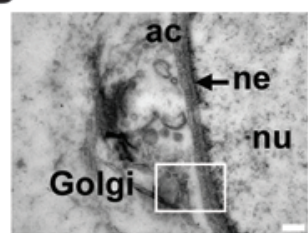

Pick1-/-
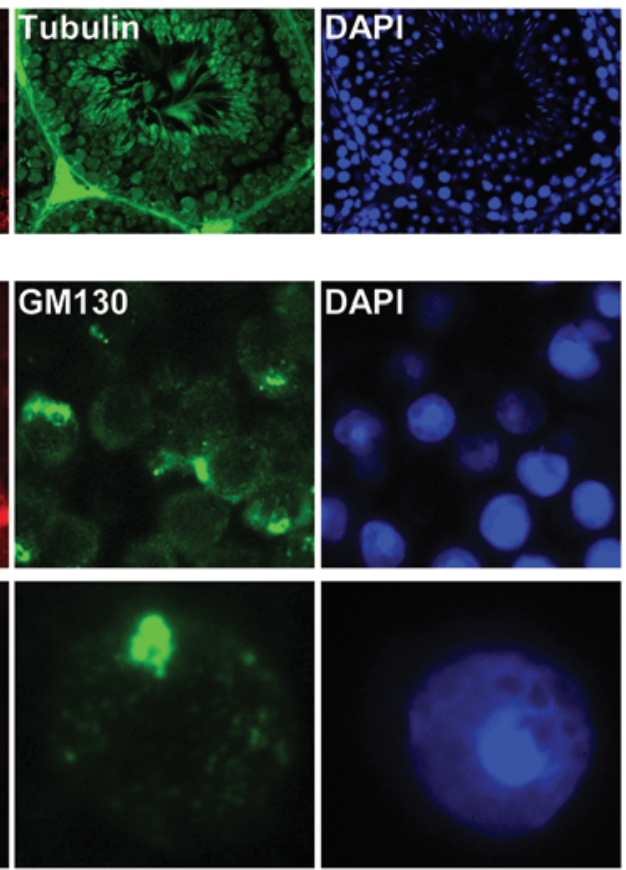
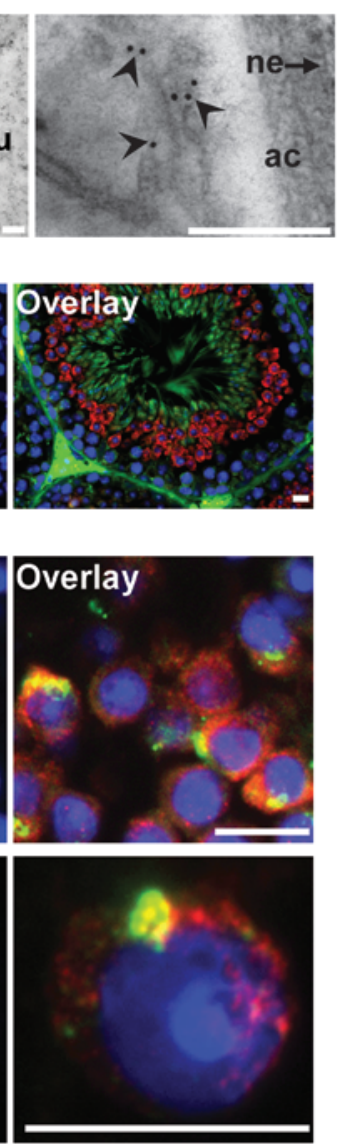

\section{Figure 3}

PICK1 is highly expressed in spermatids and localized around the Golgi apparatus. (A) DAB staining of testis sections from Pick $1^{+/+}$and Pick $1^{-/}$ mice. PICK1 is highly expressed in round spermatids. The background staining of Pick1-/- mice is low. (B) Triple immunofluorescence staining of PICK1, $\beta$-tubulin, and DAPI, which marks nuclei. PICK1 is mainly concentrated in the perinuclear region of round spermatids. (C) PICK1 is partially colocalized with the Golgi marker GM130 in the spermatids in both the testis sections (upper panels) and testis smear stains (lower panels). Scale bars: $10 \mu \mathrm{m}$. (D) PICK1 is located on the Golgi-derived proacrosomal granules between the Golgi apparatus and the acrosome (ac). The arrowheads indicate gold particles labeling PICK1. The arrow indicates the nucleus envelope (ne). The right panel shows a higher-magnification view of the boxed region in the left panel. Scale bar: $200 \mathrm{~nm}$.
(Figure 2B). We measured the weight of the testes and normalized it to the body weight of the mice. We found that the testes of Pick $1^{-1-}$ mice were significantly lighter than those of both wild-type and $\mathrm{Pick}^{+/-}$mice (Figure 2C). Moreover, the testes of Pick ${ }^{+/-}$mice were slightly lighter than those of wild-type mice.

From the H\&E staining of sectioned testes, we found that the seminiferous tubules in Pick $1^{-/-}$mice are generally smaller than those in wild-type mice (Figure 2E). We measured the diameters of round seminiferous tubules and found that they were significantly smaller in Pick $1^{-/-}$mice $(181.3 \pm 4.2 \mu \mathrm{m})$ than in the wildtype $(203.7 \pm 1.1 \mu \mathrm{m})$ and Pick $1^{+/-}(206.2 \pm 5.2 \mu \mathrm{m})$ mice (Figure 2D). The layers of cells in the seminiferous tubules were largely preserved in Pick1 $1^{-/-}$mice, with no detectable changes in the spermatogonia (Figure 2E, white arrows), spermatocytes (white arrowheads), and round spermatids (black arrows). However, the sperm near the center of the lumen of Pick $1^{-/-}$mice had round nuclei (Figure $2 \mathrm{E}$, black arrowheads), and there were fewer mature sperm in the lumen. This suggests that the abnormalities of the sperm of Pick $1^{-1-}$ mice likely occur in the transition from round spermatids to mature sperm during spermiogenesis.

PICK1 localizes to the Golgi-derived vesicles in the round spermatids. To understand how a deficiency in PICK1 results in abnormal sperm, we determined the localization of PICK1 in mouse testes. We found PICK1 to be highly expressed in the round spermatids of the seminiferous tubules, as revealed by immunohistochemical analysis using diaminobenzidine tetrahydrochloride (DAB) as the substrate (Figure 3A). This was confirmed by immunofluorescence staining, which showed that PICK1 is localized in the perinuclear regions of round spermatids (Figure 3B). High magnification revealed that PICK1 is concentrated in one end of the cytoplasm and partially overlaps with GM130, an established marker of the Golgi apparatus (Figure $3 \mathrm{C}$ ). This suggests that PICK1 might be involved in Golgi-related vesicle trafficking in spermatids. To confirm this, we labeled PICK1 with gold particles and examined its distribution in the round spermatids under a transmission electron microscope (TEM). PICK1 was found to be enriched in the region between the Golgi apparatus and the acrosomes in the round spermatids (Figure 3D). Particularly, PICK1 was associated with Golgi-derived proacrosomal granules (Figure 3D, arrowheads).

PICK1 deficiency leads to malformation of acrosome. Since an acrosome is formed by proacrosomal granules derived from the Golgi apparatus, the localization of PICK1 to Golgi-derived vesicles prompted us to examine the relationship between PICK1 and acrosome formation. We used an antibody against the acrosome matrix protein sp56 as a marker to label the acrosome at different stages of spermiogenesis. In the early stages of spermiogenesis, i.e., in Golgi phase spermatids, PICK1 (Figure 4A, arrowheads, red signal) localized near or partially overlapped with the acrosome (Figure 4A, arrows, green signal). In the cap phase, acrosomes grew into caplike structures (Figure 4A, arrows). PICK1 remained close to the acrosomes but began to migrate to other regions around the nuclei (Figure 4A, arrowheads). PICK1 further separated from the acrosomes as spermiogenesis progressed. In the acrosome phase, while acrosomes formed hook-like structures and moved toward one end 
A
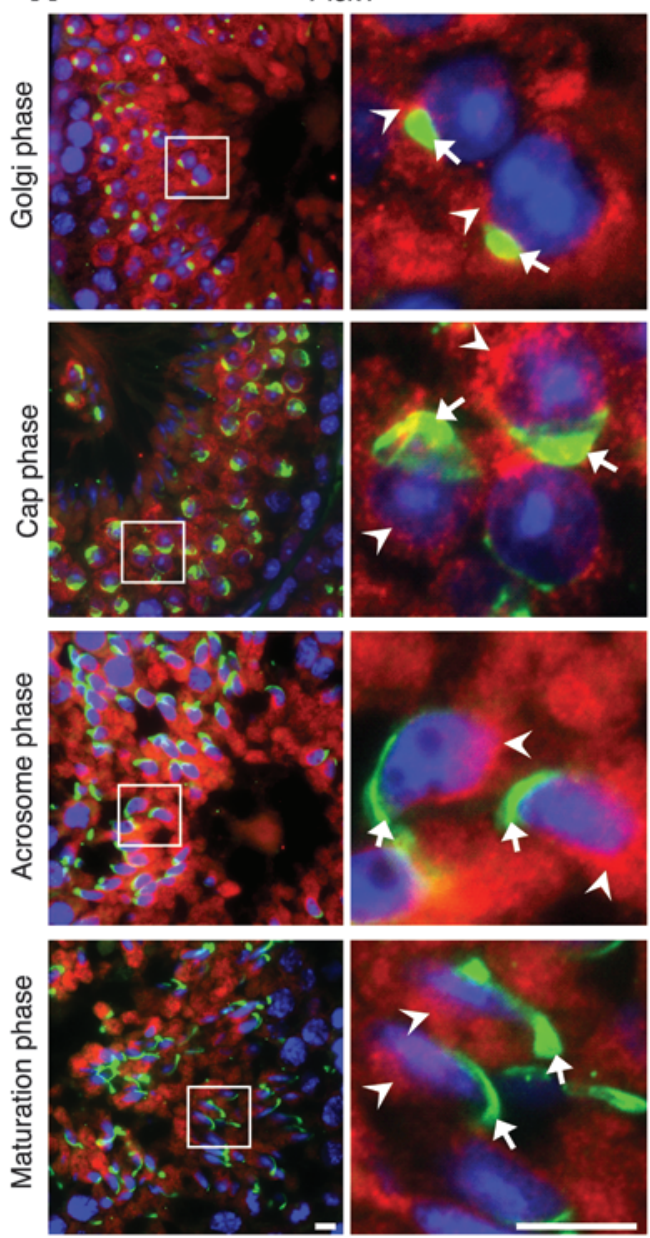

B
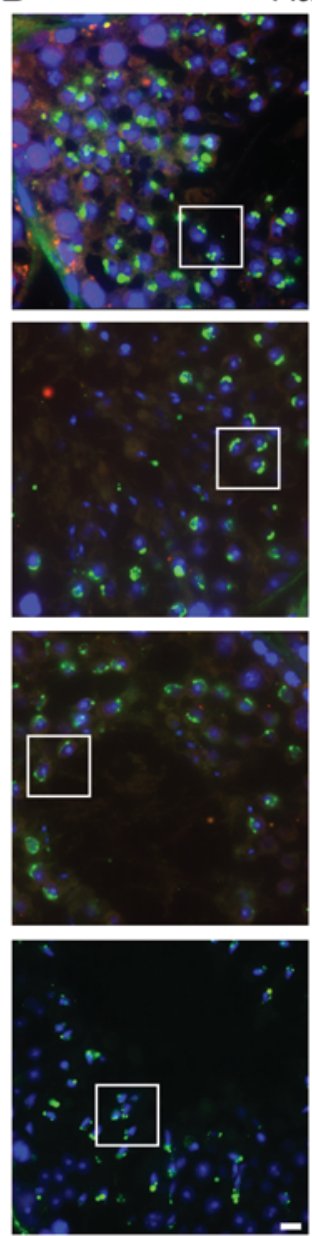

Pick $1^{-1-}$
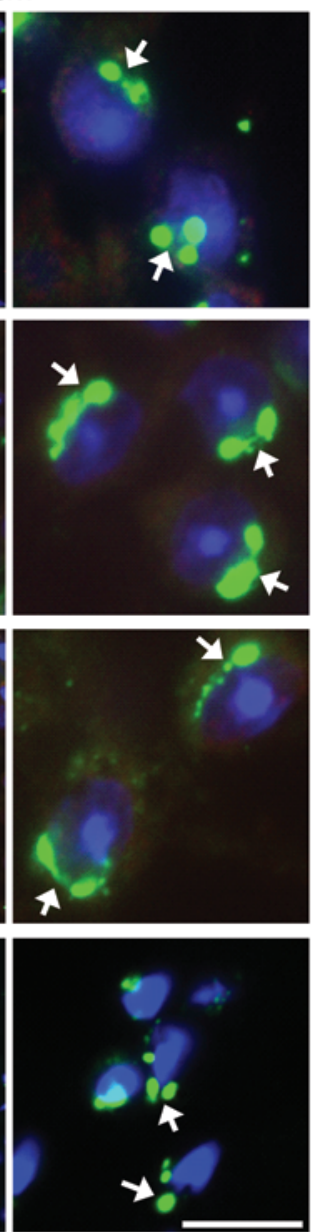

\section{Figure 4}

Acrosome formation is disrupted in Pick1-/- mice. Immunostaining of testis sections. PICK1 (red) and acrosome (green) were labeled by a guinea pig antibody against PICK1 and a mouse antibody against sp56. Nuclei were marked by DAPI (blue). Four phases of spermiogenesis are shown in progressive order. (A) Images from wild-type mice. PICK1 signals (arrowheads) are close to the acrosomes (arrows) in the Golgi phase and the cap phase but move to opposite ends of the nucleus in the acrosome and maturation phases. Acrosomes grow from single granules in the Golgi phase to caps covering the heads of the nuclei in the cap phase to the crescent moon-shaped structures at one pole of the nuclei in the acrosome and maturation phases. (B) Images from Pick1-knockout mice. PICK1 signals are notably missing in testes from Pick $1^{-/-}$mice. There are multiple sp56-positive structures in spermatids of Pick $1^{-/-}$mice throughout spermiogenesis. The morphology of the nuclei of the spermatids of Pick $1^{+/+}$and Pick $1^{-/-}$mice was similar in the Golgi and cap phases. However, while the nuclei of Pick $1^{+/+}$mice become elongated in the acrosome and maturation phases, those of Pick $1^{-1-}$ mice remain round-shaped. Scale bars: $10 \mu \mathrm{m}$. Right panels show higher-magnification views of the boxed regions in the left panels.

of the nuclei (Figure 4A, arrows), PICK1 moved to the opposite end (Figure 4A, arrowheads). In the maturation phase, PICK1 completely moved to the end opposite the acrosomes, and, at the end of spermiogenesis, PICK1 was removed in the residue body together with most of the other cytosolic components and was absent from the mature sperm (Figure 4A and data not shown).

We observed defects in acrosome formation as early as in the Golgi phase during spermiogenesis in Pick1 $1^{-/}$mice. In the Golgi phase, proacrosomal granules derived from the Golgi apparatus fused into a single acrosomal structure attached to one end of the nucleus in wild-type mice (Figure 4A, arrows). In contrast, phase (Figure 5, A and B, arrows) There was no apparent cap structure in Pick1/- spermatids in the following cap phase (Figure 5B). Instead, several large acrosomal granules attached directly to the nuclear membrane at various sites (Figure 5B, arrows). The fragmented acrosome persisted in all subsequent stages of spermiogenesis (Figure 5B, arrows). It is also evident that the elongation of the nuclei of spermatids in Pick1-/- mice was impaired in the late stages of spermiogenesis, which led to roundshaped nuclei (Figure 5B). We also examined the sperm in the epididymis. While the mitochondria wrapped around the axoneme properly in the sperm of wild-type mice, they were aggregated and wrapped around the head of the sperm in Pick1 ${ }^{-/}$mice (Figure 5C). 
A
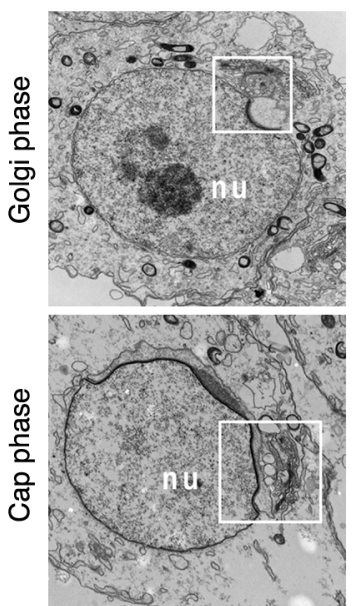

$k 1^{+/ 4}$
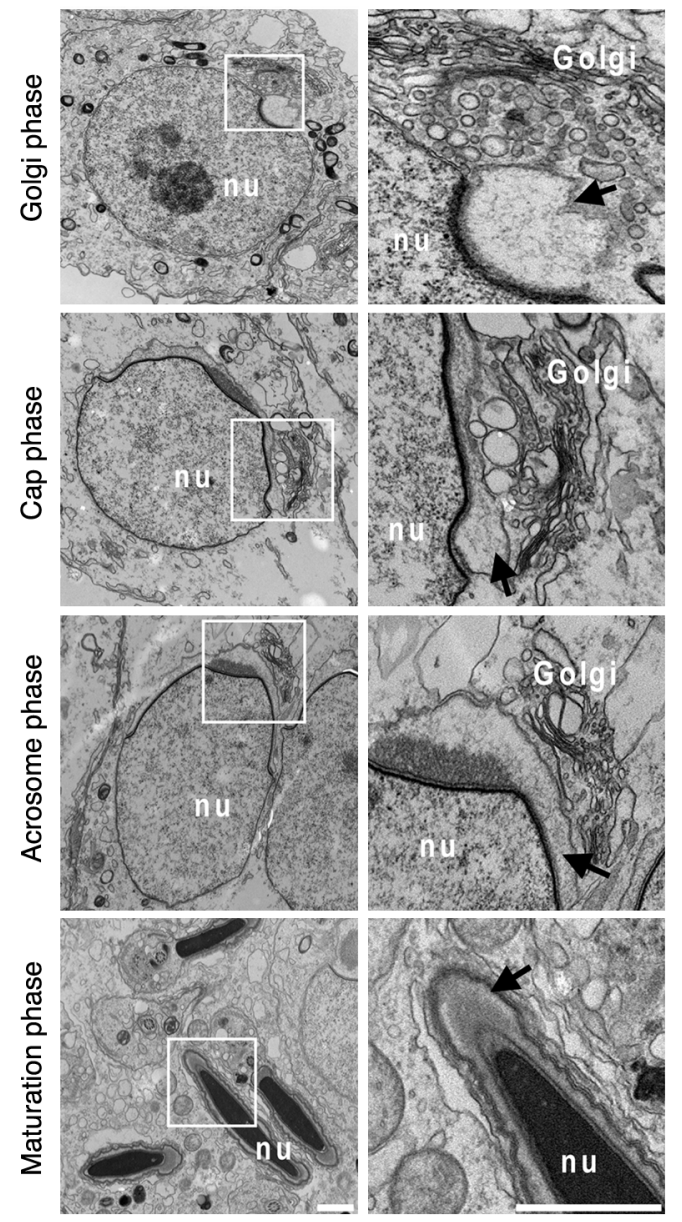

C
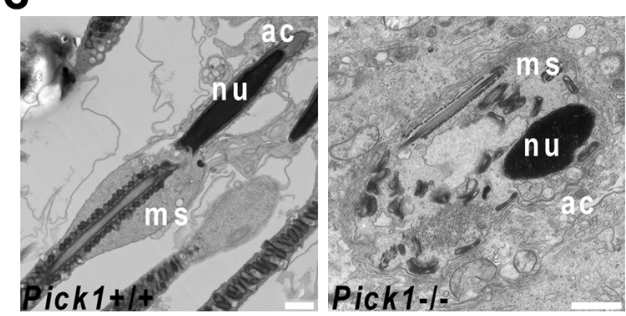

B
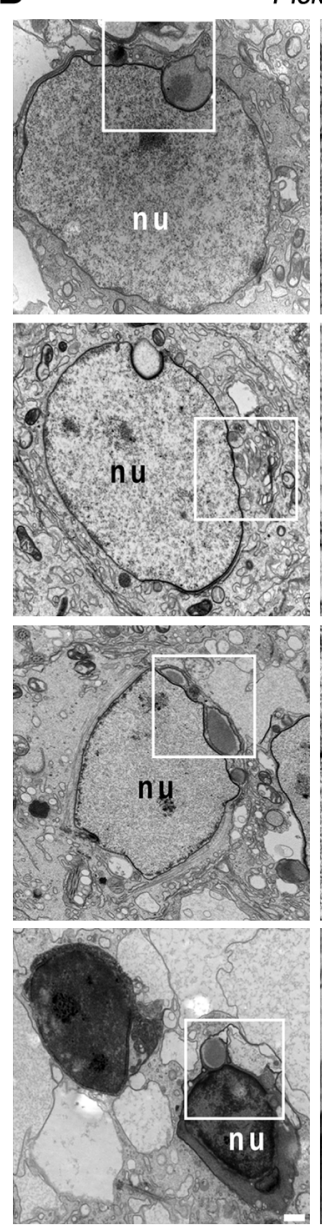

Pick1-1-
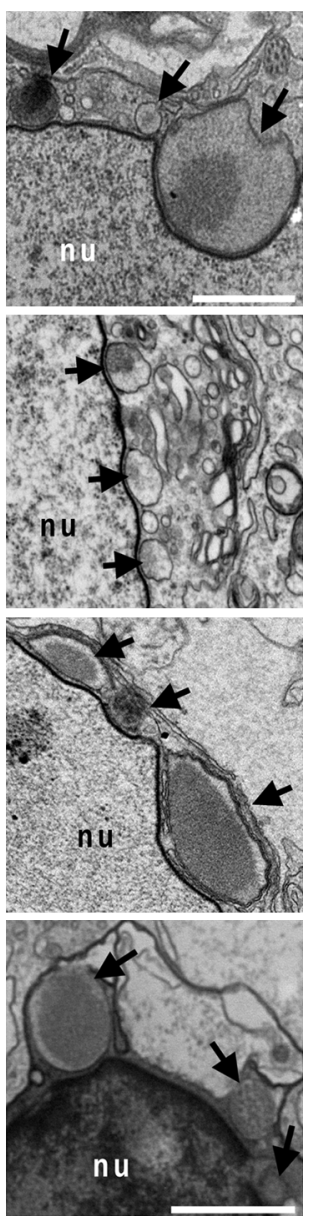

\section{Figure 5}

TEM study of acrosome formation in Pick $1^{-/-}$mice. TEM images of spermatids showing 4 phases of spermiogenesis. (A) In the wildtype mice, a large number of Golgiderived proacrosomal granules are present in the Golgi phase, when a single acrosome granule can be seen attached to the nuclear envelope. The acrosome flattens and grows to form a cap at one end of the nucleus in the cap phase. The acrosome becomes extended along the nuclear envelope in the subsequent acrosome and maturation phases. The nucleus starts to elongate in the acrosome phase and becomes hook-shaped in the maturation phase. (B) In Pick $1^{-/-}$mice, multiple acrosomal vesicular structures are seen in all phases of spermatids, as indicated by the arrows. The nuclei of spermatids of Pick1-1- mice failed to elongate and remain round in the maturation phase. Right panels show higher-magnification views of the boxed regions in the left panels. (C) Sperm from the caput epididymis of Pick1+/+ and Pick $1^{-1-}$ mice. While mitochondria wrap around the axoneme in the midpiece of sperm from Pick $1^{+/+}$ mice, they aggregate around the deformed nucleus in Pick $1^{--}$mice. The deformed acrosome is also shown. Scale bars: $1 \mu \mathrm{m}$. ms, mitochondria sheath.
PICK1 interacts with GOPC and CK2 $\alpha^{\prime}$. To gain a more comprehensive understanding of PICK1's function in acrosome formation and how PICK1 deficiency leads globozoospermia, we searched the literature for knockout mice with testicular abnormalities similar to those of Pick1-knockout mice. We found that mice with deficiencies in 5 other proteins have phenotypes similar to that of Pick1-knockout mice. These include the primary catalytic subunit of protein kinase $2\left(\mathrm{CK} 2 \alpha^{\prime}\right)$, a serine/threonine kinase regulating the cell cycle and many other cellular functions (24); Hrb (also called RAB or hRIP), an HIV-1 Rev binding protein involved in viral replication (25); GOPC, a Golgi-associated PDZand coiled-coil motif-containing protein that may be involved in vesicle trafficking (26); and ZPBP1, a zona pellucida binding protein, and its paralog ZPBP2 (27). To investigate the relationships among these genes, we performed yeast two-hybrid assays to test the protein-protein interactions of these genes. Interest- ingly, we found that PICK1 binds to GOPC and CK2 $\alpha^{\prime}$ (Table 1). We did not observe any interaction of PICK1 with Hrb, ZPBP1, or ZPBP2, nor did we detect any interactions among GOPC, CK2 $\alpha^{\prime}$, $\mathrm{Hrb}$, ZPBP1, and ZPBP2. GOPC and PICK1 self-interactions were also detected in the assay (Table 1 ), in agreement with previous reports that GOPC and PICK1 form homo-oligomers (4, 28-30). We quantified the interaction strength using a liquid $\beta$-galactosidase assay and found that PICK1's interactions with GOPC and CK2 $\alpha^{\prime}$ were significantly stronger than its interactions with the vector control and other noninteracting proteins (Figure 6A). The interactions between PICK1 and GOPC and between PICK1 and CK2 $\alpha^{\prime}$ were further confirmed by coimmunoprecipitation from mammalian cells. In HEK293T cells cotransfected with GOPC and PICK1 or CK2 $\alpha^{\prime}$ and PICK1, GOPC and CK2 $\alpha^{\prime}$ were robustly coimmunoprecipitated with PICK1 by an anti-PICK1 antibody (Figure 6, B and C). 
Table 1

Interactions among globozoospermia-related proteins

\begin{tabular}{ccccccc}
\hline & \multicolumn{7}{c}{ BD } & & BD vector \\
AD & PICK1 & GOPC & CK2 $\alpha^{\prime}$ & ZPBP1 & ZPBP2 & \\
PICK1 & + & + & + & - & - & - \\
GOPC & + & + & - & - & - & - \\
Hrb & - & - & - & - & - & - \\
AD vector & - & - & - & - & - & - \\
\hline
\end{tabular}

Interactions among proteins indicated were tested by yeast two-hybrid assays. Positive and negative interactions are designated with + and - , respectively. BD, GAL4 binding domain; AD, GAL4 activation domain.

PICK1 and GOPC partially colocalize in the Golgi region and may coordinate formation of trafficking vesicles. GOPC has been reported to be localized in the Golgi apparatus $(31,32)$. We examined the localization of PICK1 and GOPC in spermatids by immunofluorescence. In round spermatids, the PICK1 signal partially overlapped with the GOPC signal in the Golgi region, which was marked by GM130 (Figure 7A). To evaluate the relationships among PICK1, GOPC, and the Golgi apparatus, we treated isolated germ cells from adult rat testes with brefeldin A (BFA), a drug that blocks vesicle budding from Golgi apparatus. A brief (5-minute) treatment with BFA disrupted the signal of GM130, but not TGN38, a marker for the trans-Golgi network (data not shown). This result is in agreement with previous observations that brief BFA treatment disrupts the cis-Golgi but not the trans-Golgi network (33). Interestingly, a 5-minute BFA treatment led to concentration of both PICK1 and GOPC in the trans-Golgi network, as marked by the TGN38 signal, and a reduction in PICK1 and GOPC granules outside the Golgi apparatus (Figure 7B). This is likely because BFA blocked vesicle budding and caused retention of PICK1 and GOPC in the transGolgi network. This result indicates that PICK1 and GOPC are both involved in the budding of vesicles from the trans-Golgi network. To investigate the potential functional significance of the PICK1-GOPC interaction, we cotransfected PICK1 and GOPC into HEK293T cells. Expression of PICK1 in HEK293T cells is known to form small clusters that may represent trafficking vesicles (11). When GOPC was expressed together with PICK1, they partially colocalized in the perinuclear region, and the number of PICK1

\section{Figure 6}

PICK1 interacts with GOPC and CK2 $\alpha^{\prime}$, but not with Hrb, ZPBP1, or ZPBP2. (A) Yeasts cotransformed with PICK1 and other CDNA as indicated were grown on liquid selective medium, and $\beta$-galactosidase activity was measured using ONPG as the substrate. Data are presented as mean $\pm \mathrm{SEM} ; n=4 ;{ }^{* \star} P<0.01$ compared with vector control, as determined by Student's $t$ test. (B) GFP-GOPC transfected into HEK293T cells together with myc-PICK1 or the vector control. PICK1 was immunoprecipitated by an anti-PICK1 antibody. Anti-myc antibody (upper panel) or anti-GFP antibody (lower panel) was used for Western blotting. Expression of GFP-GOPC and myc-PICK1 in 293T cells is indicated in the lanes labeled "Input." The immunoprecipitation products indicate that GFP-GOPC was pulled down in the presence only of PICK1 but not the vector control. (C) GFP-CK2 $\alpha^{\prime}$ and mycPICK1 or the empty myc vector were cotransfected into 293T cells and immunoprecipitated as described in B. Similarly, CK2 $\alpha^{\prime}$ was coimmunoprecipitated with PICK1 specifically. clusters significantly increased (Figure 7, C and D). Some of the PICK1-positive clusters also colocalized with GOPC signals (Figure $7 \mathrm{C}$, arrows). This observation suggests that GOPC coordinates with PICK1 to facilitate vesicle formation.

PICK1 binds to liposomes via its BAR domain, and this lipidbinding capability correlates with PICK1's ability to form clusters in HKE293T cells (11). The increased number of PICK1 clusters upon coexpression with GOPC prompted us to examine whether GOPC could also bind to liposomes. Purified glutathione $S$-transferase-fused (GST-fused) GOPC protein was incubated with liposomes and then subjected to centrifugation. Proteins associated with the liposomes will come down with liposomes and show up in the pellet. We found that GOPC indeed binds to the liposomes, as GST-GOPC was found in the pellet only in the presence of liposomes (Figure 7E). This suggests that by interacting with PICK1 and binding to liposomes, GOPC may facilitate PICK1's association with trafficking vesicles and lead to the increased formation of PICK1 clusters (Figure 8C).

Increased apoptosis in the seminiferous tubules of Pick1-/- mice. The smaller testes, reduced sperm count, and smaller seminiferous tubules of Pick1 $1^{-/-}$mice point to potential loss of germ cells in these mice. To evaluate this possibility, we performed TUNEL staining on the testes and found a significant increase in apoptosis in the seminiferous tubules of Pick $1^{-/}$mice (Figure 8A). The percentage of TUNEL-positive cells in the seminiferous tubules increased from $0.4 \%$ in wild-type mice to $2.5 \%$ in ick $^{-/-}$mice (Figure $8 \mathrm{~B}$ ). More importantly, while the apoptotic cells in wild-type mice were found to be mainly spermatogonia, a large number of the apoptotic cells in Pick $1^{-1}$ mice appeared to be spermatids (Figure 8A, arrows), suggesting that an increase in apoptosis occurs in the late stages of spermatogenesis. The increase in apoptosis is similar to that found in mice deficient in CK2 $\alpha^{\prime}$ (24), suggesting that PICK1 and CK2 $\alpha^{\prime}$ may function together. This result, together with our finding that PICK1 interacts with CK2 $\alpha^{\prime}$, support the notion that PICK1 and CK2 $\alpha^{\prime}$ work together in spermiogenesis.

\section{Discussion}

Acrosomes are formed by the fusion of proacrosomal granules derived from the trans-Golgi network (21-23). The molecular mechanisms underlying the budding, transportation, and fusion of proacrosomal granules to the eventual formation of acrosomes are largely unknown. In this study, we found that deficiency of
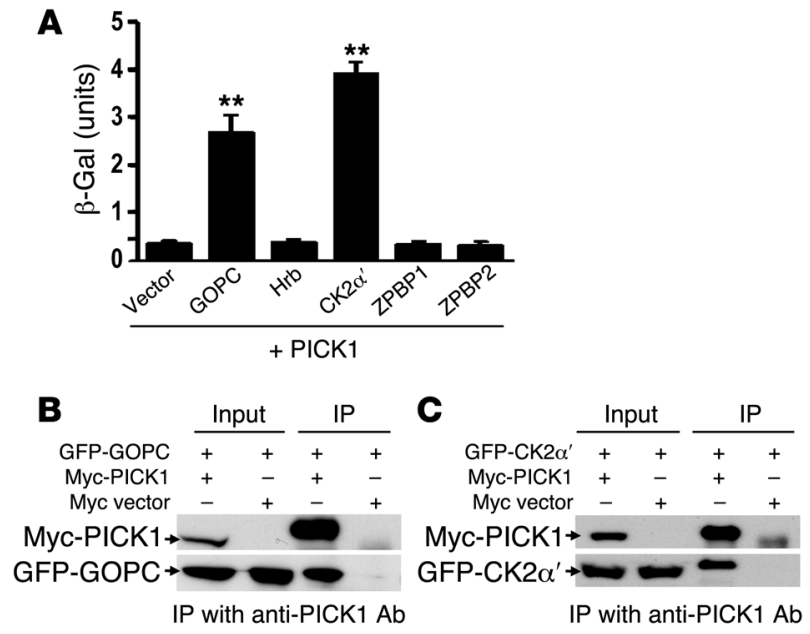
A
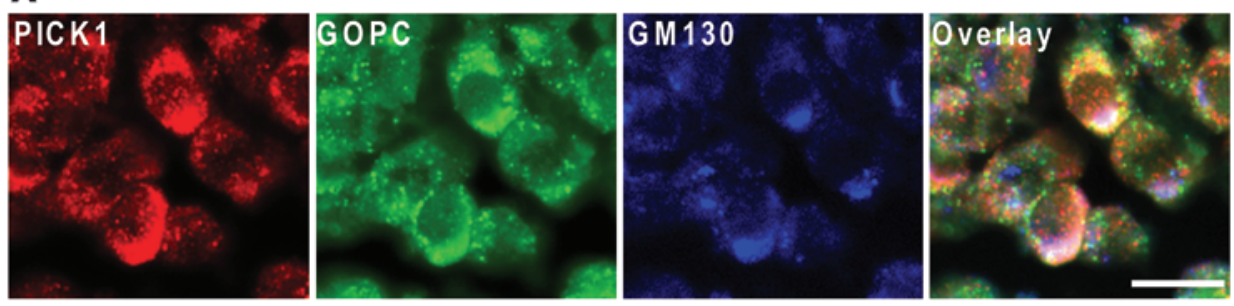

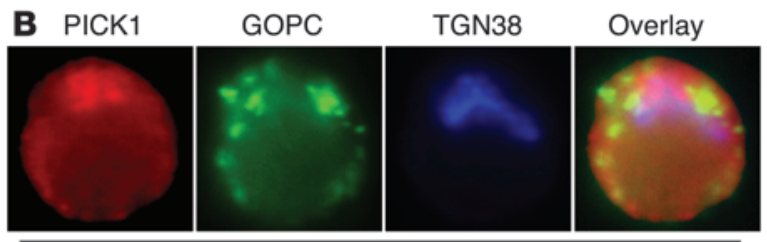

Control

C
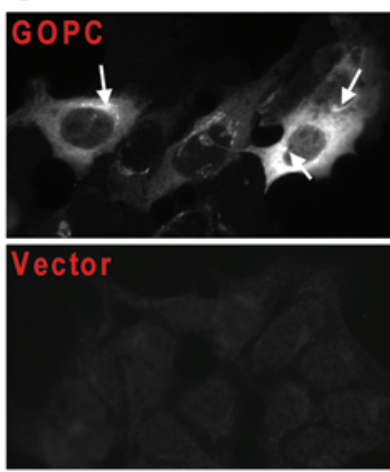

D

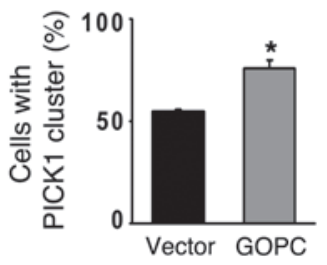

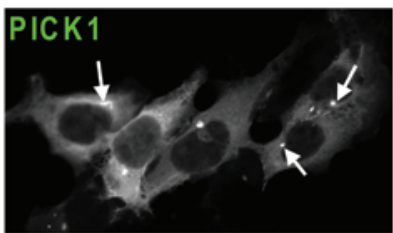
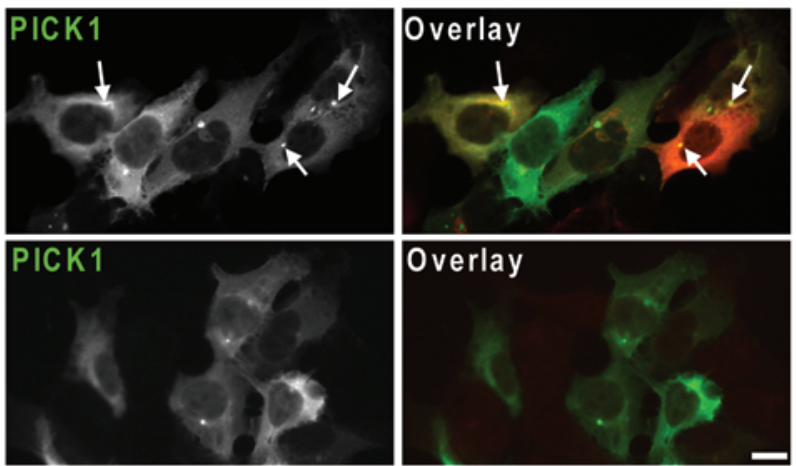

E

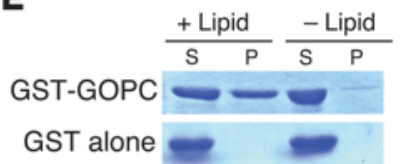

\section{Figure 7}

PICK1 and GOPC partially colocalize around the Golgi apparatus, and GOPC increases PICK1 clusters. (A) Immunofluorescence staining showed that PICK1 (red) and GOPC (green) are both expressed in the perinuclear region on the round spermatids and they partially colocalize with the Golgi marker GM130 (blue). (B) In the rat testis smear, $5 \mathrm{ng} / \mu \mathrm{l}$ BFA treatment for 5 minutes leads to better colocalization of PICK1, GOPC, and TGN38, as indicated by the arrows. (C) GFP-PICK1 and myc-GOPC or the empty myc vector were cotransfected into $293 \mathrm{~T}$ cells. GOPC increased the number of PICK1 clusters, and some of these clusters overlapped with the GOPC signal, as indicated by the arrows. (D) GOPC increases the percentage of HEK293T cells with PICK1 self-clusters to $76.1 \% \pm 3.8 \%$ from $55.0 \% \pm 0.8 \%$ in the vector control group (mean \pm SEM; $n=3$ experiments; $\left.{ }^{*} P<0.05\right)$. (E) GST-GOPC was mixed with liposome and subjected to high-speed centrifugation. Equal amounts of pellet and supernatant were loaded and resolved by SDS-PAGE. GOPC was found to associate with liposomes and appeared in the pellet (top). In the control experiments, GOPC was not found in the pellet without liposomes, and GST itself did not bind to liposomes. Scale bars: $10 \mu \mathrm{m}$.

PICK1 in mice leads to male infertility due to reduced sperm count and severely impaired sperm motility. The major defects in the sperm are the malformation of the acrosomes, round nuclei, and abnormal arrangement of mitochondrial sheaths, which are also prominent features of the human disease globozoospermia (20). PICK1 protein is highly expressed in round spermatids and localizes to the vesicles between the Golgi apparatus and the acrosomes. Furthermore, blocking vesicle budding from the Golgi apparatus by BFA caused retention of PICK1 in the trans-Golgi network. This suggests that PICK1 may take part in vesicular trafficking from the trans-Golgi network to the acrosomes, such as in the budding of proacrosomal granules, sorting of cargo proteins, or transporting proacrosomal granules to form mature acrosomes (Figure $8 \mathrm{C}$ ). The fact that PICK1 is not present in mature acrosomes suggests that PICK1 may be removed from proacrosomal granules at a certain stage and be recycled for multiple rounds of vesicle trafficking.

While one of the earliest defects in Pick1-knockout mice is fragmentation of the acrosomes, PICK1 is unlikely to be directly involved in the fusion of proacrosomal granules. Neither the PDZ domain nor the BAR domain, the 2 major domains of PICK1, have been implicated in membrane fusion. In fact, the BAR domain is believed to be involved in the budding of vesicles by actively bend- 

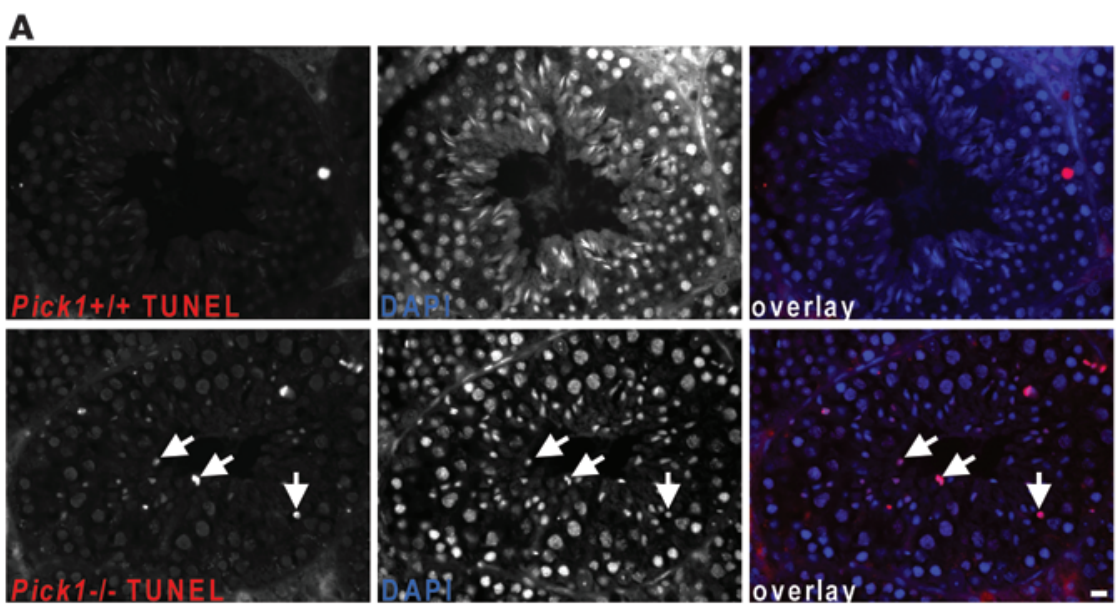

B

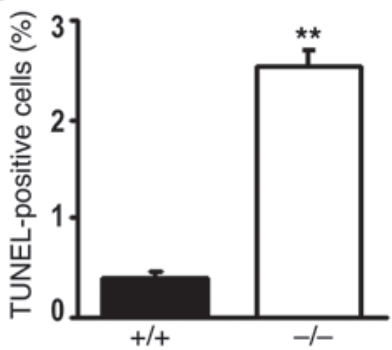

C

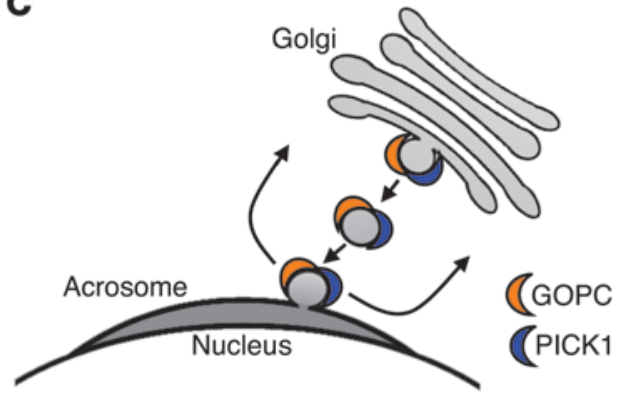

\section{Figure 8}

Increased apoptosis in the seminiferous tubules of Pick 1-l- mice. (A) TUNEL staining of the testis sections. Only a few apoptotic spermatogonia, labeled with red fluorescence, are seen in the seminiferous tubules of Pick $1^{+/+}$mice. In contrast, far more apoptotic germ cells, many of them spermatids, can be found in the seminiferous tubules of $P i c k 1^{-1-}$ mice, as indicated by the arrows. Scale bar: $10 \mu \mathrm{m}$. (B) Quantification revealed a significant increase in apoptotic cells in the seminiferous tubule of Pick $1^{-/}$mice. Data are presented as mean TUNEL-positive cells $/ 100$ cells \pm SEM; $n=30$ tubules; ${ }^{\star \star} P<0.01$. (C) Model illustrating PICK1's role in acrosome formation. PICK1 and GOPC facilitate formation of trafficking vesicles from the Golgi apparatus to the acrosome. Both of them are removed from mature acrosomes and are possibly recycled for multiple rounds of vesicle trafficking. ing lipid membranes or sensing membrane curvature $(17,34)$. This suggests that PICK1 may be involved in the budding of proacrosomal granules from the trans-Golgi network. A failure to bud proacrosomal granules may divert acrosomal matrix proteins, such as sp56, into other cellular compartments, and this may lead to fragmentation of the acrosomes. An alternative explanation is that the lack of PICK1 may indirectly lead to a deficiency in fusion of proacrosomal granules. Vesicle fusion requires properly assembled fusion machinery on both the vesicle and target membranes (35). The PDZ domain of PICK1 is known to bind membrane proteins and to regulate their trafficking (2). A deficiency in PICK1 could therefore result in the mis-sorting of membrane proteins to proacrosomal granules. Some of these membrane proteins could be part of the fusion machinery, such as Hrb or certain soluble NSF attachment protein receptor (SNARE) proteins. The lack of these fusion components eventually leads to abnormal fusion of proacrosomal granules and fragmented acrosomes.

The findings that PICK1 interacts with GOPC and that deficiency of either PICK1 or GOPC leads to globozoospermia suggest that they may function together. This notion is further supported by the fact that both PICK1 and GOPC are enriched in the Golgi region. The findings that GOPC binds to liposomes and enhances PICK1's capability to form clusters in cells and that BFA treatment retains both PICK1 and GOPC at the trans-Golgi network suggest that GOPC may coordinate with PICK1 in the formation of proacrosomal granules from the trans-Golgi network (Figure 8C). It is interesting to note that both PICK1 and GOPC were reported to regulate AMPA receptor trafficking in neurons $(2,36)$, suggesting that PICK1 and GOPC might work together in the same trafficking machinery in neuronal cells as well. It should also be noted that while PICK1 and GOPC overlap around the Golgi region, they tend to separate when moving away from the Golgi apparatus. This suggests that while PICK1 and GOPC may coordinate in the early formation of Golgi-derived vesicles, their roles in subsequent vesicular trafficking could be different. This may explain some minor differences between Pick1- and Gopc-knockout mice. For example, there is a significant reduction in the number of sperm in Pick1-knockout mice (Figure 1A), but such reduction was not observed in Gopc-knockout mice (26).

In addition to GOPC, PICK1 also interacts with CK2 $\alpha^{\prime}$, whose deficiency also leads to malformation of the acrosome and globozoospermia $(24,26)$. The fact that both PICK1 and CK2 $\alpha^{\prime}$ deficiencies lead to apoptosis in germ cells suggests that they may function together. However, how they function together remains an open question. Our preliminary data indicate that CK2 $\alpha^{\prime}$ does not affect the localization or clustering of PICK1 (our unpublished observations). Other possibilities, for example, that PICK1 regulates the kinase activity or location of CK2 $\alpha^{\prime}$, need to be tested in the future. The finding that PICK1 does not interact with Hrb, ZPBP1, and ZPBP2 but that their deficiencies lead to similar phenotypes suggests that these proteins may be involved in different steps of acrosome formation. For example, Hrb and ZPBPs could be transported as cargo by PICK1-mediated trafficking of proacrosomal granules. The identification of additional genes whose products are required for the formation of acrosomes and the elucidation of their functional relationships may provide us with a more complete picture to understand the molecular mechanism of acrosome formation as well as vesicular trafficking in general.

\section{Methods}

cDNA cloning and protein purification. All PICK1 constructs used in this study were described previously (11). The GOPC cDNA clones were gifts from 
Z. Yue (Mount Sinai School of Medicine, New York, New York, USA) and I.G. Macara (University of Virginia, Charlottesville, Virginia, USA) (28, 37). They were subcloned in-frame into PEGFP-C1 and PGEX4T2 vectors using BamHI and into pDBLeu and pPC86 vectors using SalI/NotI sites. CK2 $\alpha^{\prime}, \mathrm{Hrb}$, ZPBP1, and ZPBP2 cDNAs were obtained from RZPD German Resource Center for Genome Research and subcloned in-frame into pEGFP-C3, pPC97 or pDBLeu, and pPC86 vectors using SalI/NotI sites. To produce fusion proteins, cDNA constructs were transformed into $E$. coli BL21 cells and induced with isopropyl- $\beta$-D-thiogalactopyranoside (IPTG). GST fusion proteins were affinity purified by glutathione Sepharose 4B (Amersham Biosciences; GE Healthcare) according to the manufacturer's instructions. Fusion protein concentrations were determined by Coomassie assays (Pierce; Thermo Scientific).

Antibodies. The guinea pig anti-PICK1 polyclonal antibody and the rabbit anti-GFP polyclonal antibody were generated as previously described (18). The mouse anti-myc antibody (9E10) and the mouse anti- $\beta$-tubulin antibody (E7) were purchased from the Developmental Studies Hybridoma Bank. The mouse antibio-GM130 antibody and the mouse anti-TGN38 antibody were purchased from BD Biosciences. The mouse anti-sp56 antibody was from QED Bioscience Inc. The mouse anti-cytochrome oxidase subunit I was from Molecular Probes (Invitrogen). The rabbit anti-GOPC antibody was from Abcam. The rhodamine red X-conjugated secondary antibody was from Jackson ImmunoResearch Laboratories Inc. The Alexa Fluor 488- and Alexa Fluor 647-conjugated secondary antibodies were from Molecular Probes (Invitrogen). The HRP-labeled secondary antibody was purchased from Amersham Bioscience (GE Healthcare).

Epididymal sperm count and morphology classification. The number of epididymal sperm was determined by the method described in ref. 38. Briefly, the cauda epididymis was dissected from adult mice. Sperm were allowed to exude from incisions of the cauda epididymis for 30 minutes at $37^{\circ} \mathrm{C}$ under $5 \% \mathrm{CO}_{2}$. The incubated sperm extract medium was then diluted to $1: 500$ and transferred to the hemocytometer for counting. Unfixed sperm was spread on precoated slides for morphological observation or immunostaining. Deformities were classified according to ref. 38. The use of animals was approved by the Animal Research Panel of the Committee on Research Practice of the Hong Kong University of Science and Technology.

Histology. Adult male C57BL/6 mice were perfused with $10 \%$ neutral buffered formalin through the left ventricle. The testes and cauda epididymis were dissected, post-fixed, dehydrated through a graded ethanol series, and then embedded in paraffin. Sections $(5 \mu \mathrm{m})$ were cut on a microtome (Shandon Finesse; Thermo Fisher Scientific). The diameters of the round sections of seminiferous tubules were measured from H\&Estained testis paraffin sections using MetaMorph 7.0 software (Universal Imaging; Molecular Devices).

Testis smears preparation. The testis smears were prepared according to ref. 39. Briefly, adult male Sprague-Dawley rat or C57BL/6 mouse testes were dissected and rinsed in enriched Krebs-Ringer bicarbonate buffer (EKRB). The testes were excised and incubated with $1 \mathrm{mg} / \mathrm{ml}$ collagenase IA (SigmaAldrich), followed by $0.25 \mathrm{mg} / \mathrm{ml}$ trypsin and $1 \mu \mathrm{g} / \mathrm{ml}$ DNase I. The lysates were then centrifuged at $500 \mathrm{~g}$ for 10 minutes at $4^{\circ} \mathrm{C}$. The pellets were washed and resuspended in EKRB supplemented with $10 \%$ FBS. The suspensions were spread on poly-L-lysine-coated coverslips and cultured in EKRB. Untreated or BFA-treated $\left(5 \mathrm{ng} / \mu \mathrm{l}, 5\right.$ minutes, $\left.37^{\circ} \mathrm{C}\right)$ cells were further immunolabeled.

Immunohistochemistry. The testis paraffin sections were deparaffinized and rehydrated. Then, antigen retrieval was carried out as described in ref. 40. Immunostaining was carried out as previously described (18). Briefly, the samples were blocked, permeabilized, then incubated with primary antibody in $3 \%$ normal donkey serum at $4{ }^{\circ} \mathrm{C}$ overnight. After washing, secondary antibodies were applied for at least 1 hour at room temperature. The slides were then treated with $0.5 \mu \mathrm{g} / \mathrm{ml}$ DAPI for 15 minutes at room temperature, washed in PBS, mounted, and observed under $\mathrm{a} \times 40$ or $\times 60$ Plan Apochromat oil lens (1.4 NA; Nikon Instruments Inc.). DAB staining was carried out according to the manufacturer's recommended protocol (ABC kit; Vector Laboratories). The DAB slides were observed under $\times 20$ and $\times 40$ LC Plan F1 lens. In all the immunostaining protocols, the level of nonspecific staining was determined by omission of the incubation step with the primary antibody.

TEM. TEM was performed as previously reported (41). Briefly, testes or epididymis from adult mice were fixed with $2 \%$ glutaraldehyde or $0.25 \%$ glutaraldehyde plus $1.5 \%$ paraformaldehyde by perfusion through the left ventricle. The tissues were cut into small pieces, dehydrated, and then embedded in Spurr's resin or Lowicryl resin HM20 (Electron Microscopy Sciences). Ultrathin sections (70 nm) were cut on an Ultratome (Leica, Reichert Ultracuts). Spur sections were directly post-stained with aqueous uranyl acetate/lead citrate. HM20 sections were immunolabeled with rabbit anti-PICK1 antibody, followed by $10 \mathrm{~nm}$ gold particle-coupled secondary antibody (Electron Microscopy Sciences) and then post-stained. The observations were performed with a Hitachi H-7650 TEM operating at $80 \mathrm{kV}$. The pictures were taken with a Hitachi AMT XR-40 CCD camera.

Yeast assay. To test the interactions among PICK1, GOPC, Hrb, CK2 $\alpha^{\prime}$, ZPBP1, and ZPBP2, the corresponding DNA constructs were subcloned in-frame into PPC86 vectors that contained the GAL4 activation domain (AD) or PPC97 vectors and PDBLeu vectors that contained the GAL4 DNA binding domain (BD). The constructs were cotransformed into yeast cells (HF7c or PJ69) and grown on double-minus (lacking leucine, tryptophan) plates. Positive clones were selected and tested for growth on triple-minus plates (lacking leucine, tryptophan, histidine or adenine) to test the protein interactions. Quantitative determinations of $\beta$-galactosidase activity were performed according to ref. 42. Briefly, diploids at mid-log phase were pelleted and broken. The lysate was incubated with $o$-nitrophenyl $\beta$-D-galactopyranoside (ONPG). Time lapse $(T)$ and $\mathrm{OD}_{420}$ were recorded. $\beta$-Galactosidase activity $(A)$ expressed in $\mathrm{nmol} / \mathrm{min} / \mathrm{mg}$ was calculated according to the following formula: $A=1,000 \times \mathrm{OD}_{420} /\left(T \times V \times \mathrm{OD}_{600}\right)$, where $V$ stands for the volume of the diploid culture.

$293 T$ cell culture, coimmunoprecipitation, staining, and quantification. HEK293T cell culture, coimmunoprecipitation, and immunostaining were carried as previously described (11). Briefly, HEK293T cells were transfected by calcium phosphate coprecipitation. The cells were lysed 36-48 hours after transfection and incubated with guinea pig anti-PICK1 polyclonal antibody followed by protein A beads at $4^{\circ} \mathrm{C}$. The resin was washed, eluted with a $\times 1$ SDS sample buffer, analyzed by SDS-PAGE, and immunoblotted with primary antibodies, followed by HRP-coupled secondary antibodies (Amersham Bioscience; GE Healthcare). For immunostaining, HEK293T cells were fixed 36-48 hours after transfection, permeabilized, blocked, incubated with primary antibody for 1 hour at room temperature, and then incubated for 1 hour with fluorescent secondary antibody. Fifteen pictures were taken for each group in every experiment. The total number of double-transfected HEK293T cells and the number of transfected cells with PICK1 clusters were recorded by a researcher without knowledge of the group.

Lipid binding assay. The lipid binding assay was performed as described previously (11). Specifically, the fusion protein was incubated with brain lipid extracts and then spun at $140,000 \mathrm{~g}$ for 15 minutes at $4^{\circ} \mathrm{C}$. The supernatant and the pellet proteins were subjected to SDS-PAGE and visualized by Coomassie stain assays.

TUNEL staining. TUNEL staining was performed according to the manufacturer's recommended protocol (Roche Applied Science). Briefly, the testis paraffin sections were deparaffinized, rehydrated, and permeabilized. Each sample was incubated with the TUNEL mixture at $37^{\circ} \mathrm{C}$ for 1 hour in the dark. Positive controls were treated with $3 \mathrm{U} / \mathrm{ml}$ DNase I for $20 \mathrm{~min}$ - 
utes before TUNEL labeling, while negative controls were treated without terminal transferase during TUNEL labeling. Pictures were acquired under the same exposure time. The number of TUNEL-positive cells per 100 germ cells was determined.

Statistics. All data are presented as mean \pm SEM. Statistical differences between paired groups were measured by Student's $t$ test with paired, 2 -tailed distribution. A $P$ value of less than 0.05 was considered significant.

\section{Acknowledgments}

We thank W. Tung, K. Lai, and F. Kwok for excellent technical assistance; Z. Mao, X. Cao, and W. Chau for help in collecting the mice mating data; and D. Banfield for critical reading of the manu- script. The work described in this article was supported in part by grants from the Research Grants Council of the Hong Kong Special Administrative Region, People's Republic of China (HKUST6429/ 06M, N_HKUST605/07, HKUST6/CRF/08, and 663107).

Received for publication May 16, 2008, and accepted in revised form January 7, 2009.

Address correspondence to: Jun Xia, Department of Biochemistry, The Hong Kong University of Science and Technology, Clear Water Bay, Kowloon, Hong Kong, People's Republic of China. Phone: (852) 2358-8714; Fax: (852) 2358-1552; E-mail: jxia@ust.hk.
1. Staudinger, J., Zhou, J., Burgess, R., Elledge, S.J., and Olson, E.N. 1995. PICK1: a perinuclear binding protein and substrate for protein kinase $C$ isolated by the yeast two-hybrid system. J. Cell Biol. 128:263-271.

2. Xu, J., and Xia, J. 2007. Structure and function of PICK1. Neurosignals. 15:190-201.

3. Torres, R., et al. 1998. PDZ proteins bind, cluster, and synaptically colocalize with Eph receptors and their ephrin ligands. Neuron. 21:1453-1463.

4. Xia, J., Zhang, X., Staudinger, J., and Huganir, R.L. 1999. Clustering of AMPA receptors by the synaptic PDZ domain-containing protein PICK1. Neuron. 22:179-187.

5. Dev, K.K., Nishimune, A., Henley, J.M., and Nakanishi, S. 1999. The protein kinase C alpha binding protein PICK1 interacts with short but not long form alternative splice variants of AMPA receptor subunits. Neuropharmacology. 38:635-644.

6. Torres, G.E., et al. 2001. Functional interaction between monoamine plasma membrane transporters and the synaptic PDZ domain-containing protein PICK1. Neuron. 30:121-134.

7. Duggan, A., Garcia-Anoveros, J., and Corey, D.P. 2002. The PDZ domain protein PICK1 and the sodium channel $\mathrm{BNaC} 1$ interact and localize at mechanosensory terminals of dorsal root ganglion neurons and dendrites of central neurons. J. Biol. Chem. 277:5203-5208.

8. Hruska-Hageman, A.M., Wemmie, J.A., Price, M.P., and Welsh, M.J. 2002. Interaction of the synaptic protein PICK1 (protein interacting with $\mathrm{C}$ kinase 1) with the non-voltage gated sodium channels BNC1 (brain $\mathrm{Na}+$ channel 1) and ASIC (acid-sensing ion channel). Biochem. J. 361:443-450.

9. Dingledine, R., Borges, K., Bowie, D., and Traynelis, S.F. 1999. The glutamate receptor ion channels. Pharmacol. Rev. 51:7-61.

10. Shepherd, J.D., and Huganir, R.L. 2007. The cell biology of synaptic plasticity: AMPA receptor trafficking. Annu. Rev. Cell Dev. Biol. 23:613-643.

11. Jin, W., et al. 2006. Lipid binding regulates synaptic targeting of PICK1, AMPA receptor trafficking, and synaptic plasticity. J. Neurosci. 26:2380-2390.

12. Xia, J., Chung, H.J., Wihler, C., Huganir, R.L., and Linden, D.J. 2000. Cerebellar long-term depression requires PKC-regulated interactions between GluR2/3 and PDZ domain-containing proteins. Neuron. 28:499-510.

13. Kim, C.H., Chung, H.J., Lee, H.K., and Huganir, R.L. 2001. Interaction of the AMPA receptor subunit GluR2/3 with PDZ domains regulates hippocampal long-term depression. Proc. Natl. Acad. Sci.U. S. A. 98:11725-11730.

14. Matsuda, S., Launey, T., Mikawa, S., and Hirai, H.
2000. Disruption of AMPA receptor GluR2 clusters following long-term depression induction in cerebellar Purkinje neurons. EMBO J. 19:2765-2774.

15. Steinberg, J.P., et al. 2006. Targeted in vivo mutations of the AMPA receptor subunit GluR2 and its interacting protein PICK1 eliminate cerebellar long-term depression. Neuron. 49:845-860.

16. Gardner, S.M., et al. 2005. Calcium-permeable AMPA receptor plasticity is mediated by subunitspecific interactions with PICK1 and NSF. Neuron. 45:903-915.

17. Peter, B.J., et al. 2004. BAR domains as sensors of membrane curvature: the amphiphysin BAR structure. Science. 303:495-499.

18. Cao, M., et al. 2007. PICK1-ICA69 heteromeric BAR domain complex regulates synaptic targeting and surface expression of AMPA receptors. J. Neurosci. 27:12945-12956.

19. Pietropaolo, M., et al. 1993. Islet cell autoantigen 69 kD (ICA69). Molecular cloning and characterization of a novel diabetes-associated autoantigen. J. Clin. Invest. 92:359-371.

20. Dam, A.H., et al. 2007. Globozoospermia revisited. Hum. Reprod. Update. 13:63-75.

21. Abou-Haila, A., and Tulsiani, D.R. 2000. Mammalian sperm acrosome: formation, contents, and function. Arch. Biochem. Biophys. 379:173-182.

22. Moreno, R.D., and Alvarado, C.P. 2006. The mammalian acrosome as a secretory lysosome: new and old evidence. Mol. Reprod. Dev. 73:1430-1434.

23. Russell, L.D., Ettlin, R.A., Sinha Hikim, A.P., and Clegg, E.D. 1990. Histological and histopathological evaluation of the testis. Cache River Press. Vienna, Illinois, USA. 286 pp.

24. Xu, X., Toselli, P.A., Russell, L.D., and Seldin, D.C. 1999. Globozoospermia in mice lacking the casein kinase II alpha' catalytic subunit. Nat. Genet. 23:118-121.

25. Kang-Decker, N., et al. 2001. Lack of acrosome formation in Hrb-deficient mice. Science. 294:1531-1533.

26. Yao, R., et al. 2002. Lack of acrosome formation in mice lacking a Golgi protein, GOPC. Proc. Natl. Acad. Sci. U. S. A. 99:11211-11216.

27. Lin, Y.N., Roy, A., Yan, W., Burns, K.H., and Matzuk, M.M. 2007. Loss of zona pellucida binding proteins in the acrosomal matrix disrupts acrosome biogenesis and sperm morphogenesis. Mol. Cell. Biol. 27:6794-6805.

28. Neudauer, C.L., Joberty, G., and Macara, I.G. 2001. PIST: a novel PDZ/coiled-coil domain binding partner for the rho-family GTPase TC10. Biochem. Biophys. Res. Commun. 280:541-547.

29. Cheng, J., et al. 2002. A Golgi-associated PDZ domain protein modulates cystic fibrosis transmembrane regulator plasma membrane expres- sion. J. Biol. Chem. 277:3520-3529.

30. Staudinger, J., Lu, J., and Olson, E.N. 1997. Specific interaction of the PDZ domain protein PICK1 with the $\mathrm{COOH}$ terminus of protein kinase C-alpha. J. Biol. Chem. 272:32019-32024.

31. Charest, A., Lane, K., McMahon, K., and Housman, D.E. 2001. Association of a novel PDZ domaincontaining peripheral Golgi protein with the Q-SNARE (Q-soluble N-ethylmaleimide-sensitive fusion protein (NSF) attachment protein receptor) protein syntaxin 6. J. Biol. Chem. 276:29456-29465.

32. Yao, R., Maeda, T., Takada, S., and Noda, T. 2001. Identification of a PDZ domain containing Golgi protein, GOPC, as an interaction partner of frizzled. Biochem. Biophys. Res. Commun. 286:771-778.

33. Klausner, R.D., Donaldson, J.G., and LippincottSchwartz, J. 1992. Brefeldin A: insights into the control of membrane traffic and organelle structure. J. Cell Biol. 116:1071-1080.

34. Takei, K., Slepnev, V.I., Haucke, V., and De Camilli, P. 1999. Functional partnership between amphiphysin and dynamin in clathrin-mediated endocytosis. Nat. Cell Biol. 1:33-39.

35. Jahn, R., Lang, T., and Sudhof, T.C. 2003. Membrane fusion. Cell. 112:519-533.

36. Cuadra, A.E., Kuo, S.H., Kawasaki, Y., Bredt, D.S., and Chetkovich, D.M. 2004. AMPA receptor synaptic targeting regulated by stargazin interactions with the Golgi-resident PDZ protein nPIST. J. Neurosci. 24:7491-7502.

37. Yue, Z., et al. 2002. A novel protein complex linking the delta 2 glutamate receptor and autophagy: implications for neurodegeneration in lurcher mice. Neuron. 35:921-933.

38. WHO. 1999. WHO laboratory manual for the examination of human semen and sperm-cervical mucus interaction. Cambridge University Press on behalf of the World Health Organization. Cambridge, United Kingdom. 138 pp.

39. Bellve, A.R. 1993. Purification, culture, and fractionation of spermatogenic cells. In Methods in enzymology. P.M. Wassarman and M.L. DePampilis, editors. Academic Press. San Diego, California, USA. 84-113.

40. Hayat, M.A. 2002. Microscopy, immunohistochemistry, and antigen retrieval methods: for light and electron microscopy. Kluwer Academic/Plenum Publishers. New York, New York, USA. 355 pp.

41. Wang, J., et al. 2007. Protein mobilization in germinating mung bean seeds involves vacuolar sorting receptors and multivesicular bodies. Plant Physiol. 143:1628-1639.

42. Clontech Laboratories Inc. 2008. Yeast protocols handbook. Protocol PT3024-1; version, PR742227. www.clontech.com/images/pt/PT3024-1.pdf. 